\title{
Effects of Turbulent Magnetic Fields in Cosmic Ray Anisotropy
}

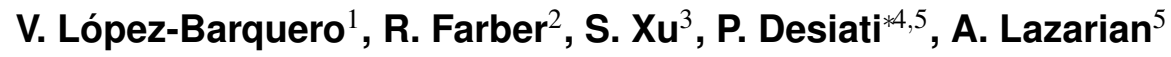
1

Department of Physics, University of Wisconsin, Madison, Wisconsin 53706, USA

2 Department of Physics and Astronomy, Wheaton College, Norton, MA 02766, USA

3 Department of Astronomy, School of Physics, Peking University, Beijing 100871, China

4 Wisconsin IceCube Particle Astrophysics Center (WIPAC), University of Wisconsin, Madison, WI 53703, USA

5 Department of Astronomy, University of Wisconsin, Madison, WI 53706, USA

\begin{abstract}
Cosmic ray anisotropy has been observed to be present in a wide energy range by a variety of experiments such as Milagro and the IceCube Observatory. However, a satisfactory explanation has been elusive for more than fifteen years now. A possible solution for the TeV-PeV cosmic ray anisotropy is the introduction of turbulent magnetic interactions on the arrival direction. We perform test particle simulations in compressible magnetohydrodynamic turbulence to study how cosmic rays' arrival direction distribution is perturbed when they stream along the local turbulent magnetic field. In this work, we discuss the effects arising from propagation in this inhomogeneous and turbulent interstellar magnetic field.
\end{abstract}

The 34th International Cosmic Ray Conference,

30 July- 6 August, 2015

The Hague, The Netherlands

\footnotetext{
* Speaker.
} 


\section{Introduction}

Above the energy range where cosmic rays are directly affected by inner heliospheric processes (see e.g. $[1,2,3]$ ), a statistically significant anisotropy was observed by a variety of experiments sensitive to different energy ranges (from tens $\mathrm{GeV}$ to a few PeV) located on and below the Earth surface, in the northern hemisphere $[4,5,6,7,8,9,10,11,12,13,14,15,16]$, and in the southern hemisphere $[17,18,19,20]$. The origin of the observed anisotropy is not understood yet, however it is reasonable to assume that it is a combination of effects correlated to the distribution of the galactic sources of cosmic rays, of the geometry and turbulent properties of the galactic magnetic field and on propagation in the interstellar magnetized plasmas. These are likely co-responsible of the complex shape of the energy spectrum as well [21]. Since we don't know where the sources of cosmic rays are and we don't know the details of the interstellar magnetic field, understanding the observations is not an easy task.

Turbulence in astrophysical plasmas can have significant effects on particle propagation, and this is the idea that we will explore in this work through integration of particle's trajectories in a magnetohydrodynamic MHD turbulent magnetic field.

\section{Turbulent magnetic field}

Test particle trajectories are integrated in a compressible sub-Alfvénic isothermal MHD turbulence in low- $\beta$ based on numerical calculations developed by [22]. In the model, turbulence is driven solenoidally in Fourier space, setting the velocity and density fields initially to unity. The calculation is performed in a cube with side $L_{b o x}=512$ grid-points, with inertial range from $L_{i n j}=204.8$ grid-points (i.e. $0.4 \times L_{b o x}$ ) down to a damping scale of $L_{m i n}=5$ grid-points.

The average magnetic field is directed along the $\mathrm{x}$-axis and turbulence is characterized by a gas-to-magnetic pressure value of $\beta \sim 0.2$ and by Alfvénic mach number $M_{A}=0.773$. Such Alfvénic mach number corresponds to the fluctuations being of the order of the mean magnetic field at the injection scale, which is in agreement to what we would have expected from the local interstellar medium. The external mean magnetic field is the only controlled parameter in this MHD model.

\section{Cosmic ray propagation}

The study is performed by integrating proton trajectories in a generic magnetic field, using the set of 6-dimensional ordinary differential equations

$$
\begin{gathered}
\frac{d \vec{p}}{d t}=q(\vec{u} \times \vec{B}) \\
\frac{d \vec{r}}{d t}=\vec{u}
\end{gathered}
$$

describing the Lorentz force and the particle velocity. $\vec{r}$ is the particle position vector and $\vec{p}$ the momemtum. For $\vec{B}$, we use one steady state realization of the magnetic field described in section 2 . The equations are integrated using the Burlisch-Stoer integration method with adaptive time step. 
At each integration step the magnetic field is interpolated using a 3D cubic spline, and integration is stopped when particles cross the border of the MHD simulation box. The choice of one specific realization of the magnetic field is justified by the fact that particle velocity is much larger than the plasma Alfvén velocity, thus induced electric fields can be neglected.

In order to study the effect of interstellar magnetic turbulence on the arrival direction distribution of cosmic rays, a large number of particles would need to be injected with randomly uniform directions on a spheric surface centered at the Earth and with radius larger than the mean free path. Unfortunately such method would result to be highly inefficient because a large fraction of the injected particles would never reach Earth. The alternative approach is typically to use the socalled "back-tracking method", where particle trajectories are integrated from Earth, with initial uniformly distributed directions backward into outer space. Since energy losses are negligible for proton particles, their energy is conserved and therefore their trajectories can be time-reversed, provided there are no collisional scattering processes and no resonant scattering which may produce chaotic trajectories. Under such general conditions Liouville Theorem guarantees that phase space distribution is conserved along particle trajectories

\section{Results}

The main question to answer is: how does the interaction with a turbulent magnetic field create the features of small scale anisotropy?

From the propagation of particles in the turbulent magnetic field, we produced maps that can be compared with the small scale features of the observations. The procedure that we performed for the creation of the maps has 5 different stages. First, we propagated the particles isotropically from the center, and let them interact with the magnetic field until they reach an outer radius. At that radius, we record the particles's position and momentum and we introduce a dipole at that distance. Using Liouville's theorem, then we backtrack the particles at Earth from that outer radius. One key factor to remember is that we are only interested in the small scale anisotropy, since this is the one that arises from the specific interaction with the turbulent magnetic field; therefore, we remove the large scale component, specifically the dipolar component of the map at Earth. For the final map, we performed a three degrees smoothing.

For the creation of the maps, we utilize Healpy [23], which divides the sphere in equal area pixels. For the present work, we use a parameter of $N_{\text {side }}=16$ which means that we have 3072 pixels in total with a mean spacing of 3.6645 degrees. Here the excesses are identified by a red color and deficits by a blue one, in a relative scale. Therefore, a pixel in which many particles pass through will be represented in a redder color than one that has only a few events. The cosmic ray permeation count is calculated counting each each particle by one, and then applying a weight. This weight is calculated by how much of the momentum of the particle at the outer radius is in the direction of the applied dipole.

In figure 1, under the assumption of the Earth's location in the interarm zone with $L_{i n j}$ of a $100 \mathrm{pc}$, we have the progression of maps when we choose different outer radius, i.e. different distances that the particles have traveled, for a particular energy of $30 \mathrm{PeV}$. From these maps, we can observe that the initial distribution of cosmic rays gets significantly distorted by the turbulent field. Once it reaches the mean free path distance, the distribution tends to stay constant. 

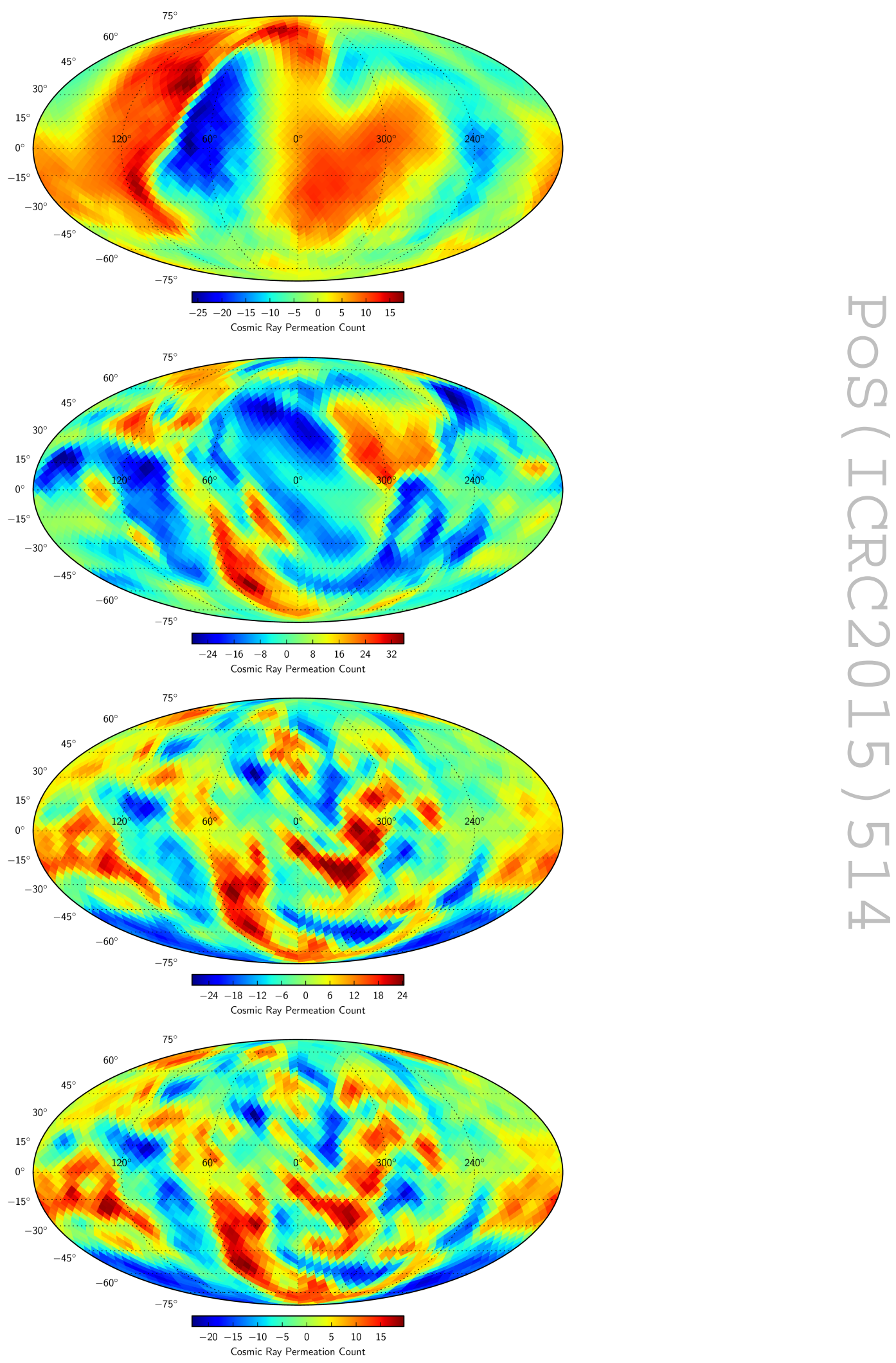

Figure 1: Progression of Arrival Distribution Maps for 30PeV Particles. Top to bottom: distance of 10pc, $20 \mathrm{pc}, 60 \mathrm{pc}$ and $90 \mathrm{pc}$. The dipole moment has been removed in the maps, and a 3 degrees smoothing has been applied. 


\section{Discussion}

We have shown how small scale anisotropy arises from the interaction of particles with the turbulent magnetic field. Specifically, how the integration of trajectories in a MHD turbulent magnetic field provides a realistic understanding of the small features present in the observations of anisotropy at Earth. The interstellar medium is in a plasma state, there the MHD equations dictate its dynamics. For this reason, a MHD turbulent magnetic field is a natural approach to study the ISM's structure, which is the main characteristic involve in this work. In our maps, the turbulent field creates very distinctive areas of higher density and deficits on the distribution of cosmic rays at a radius of the order of the injection scale. Consequently, we can see that anisotropy emerges from the propagation of these particles from a source. On the other hand, we can also interpret these results in a complementary manner, saying that the anisotropy observed at Earth will be affected by these deformations caused by the turbulent magnetic field.

\section{Conclusions}

We have explored the possibility that the local interstellar magnetic field could shape the high energy cosmic rays arrival distribution. Our results show anisotropy that arises from the interaction of CRs with the local turbulent magnetic field; therefore, local turbulent effects are crucial for the arrival of CR to the Earth. The study of how the properties of our Local Turbulent Magnetic Field may influence $\mathrm{PeV}$ cosmic ray arrival direction distribution will provide the basis of exploring the observed anisotropy, and it will open the doors for a better understanding of our local interstellar medium.

\section{References}

[1] Florinski, V, Jokipii, J. R., Alouani-Bibi, F., le Roux, J. A. 2013, Astrophys. J. 776, L37

[2] Manuel, R., Ferreira, S., \& Potgieter, M. 2014, Solar Physics 289, 2207

[3] Florinski, V., Stone, E.C., Cummings, A.C., \& le Roux, J.A. 2015, Astrophys. J. 80347

[4] Nagashima, et al. 1998, J. of Geophys. Res. 1031, 17429

[5] Hall, D.L. et al. 1999, J. of Geophys. Res. 104, 6737

[6] Amenomori, M. et al. 2005, Astrophys. J. Lett. 626, L29

[7] Amenomori, M. et al. 2006, Science, 314, 439

[8] Guillian, G. et al. 2007, Phys. Rev. D 75, 062003

[9] Abdo, A.A. et al. 2009, Astrophys. J. 698, 2121

[10] Aglietta, M. et al. 2009, Astrophys. J. 692, L130

[11] Zhang, J.L. et al. 2009, Proc. 31st ICRC, Łódź, Poland

[12] Munakata, K. et al. 2010, Astrophys. J. 712, 1100

[13] Amenomori, M. et al. 2011, Proc. 32nd ICRC, Beijing China

[14] de Jong, J. et al. 2011, Proc. 32nd ICRC, Beijing, China 
[15] Shuwang, C. et al. 2011, Proc. 32nd ICRC, Beijing China

[16] Di Sciascio, G. et al. 2013, EPJ Web Conf. 52, 04004

[17] Abbasi, R. et al. 2010a, Astrophys. J. 718, L194

[18] Abbasi, R. et al. 2011a, Astrophys. J. 74016

[19] Abbasi et al. 2012b, Astrophys. J. 746, 33

[20] Aartsen, M. et al. 2013b, Astrophys. J. 765, 55

[21] Gaisser, T.K. et al. 2013 Front. of Phys. 8-6, 748

[22] Cho, J., \& Lazarian, A. 2002, Phys. Rev. Lett. 88, 245001

[23] Gorski, K. M., Hivon, E., Banday, A. J., et al. 2005, ApJ, 622, 759 